\title{
Late Complication of Tracheostomy: Larynx-Shaped Reorganization of the Tracheal Rings or the True Larynx?
}

\author{
Jacopo Galli, Sara Giannantonio, Gaetano Paludetti \\ Institute of Otorhinolaryngology, Catholic University of the Sacred Heart, \\ “A. Gemelli” University Hospital, Rome, Italy \\ Email: sara_giannantonio@yahoo.it
}

Received May 16, 2011; revised October 13, 2011; accepted December 3, 2011

\begin{abstract}
Tracheostomy is a well-established, commonly used surgical procedure, whose early and late complications are widely described in literature. Some of them remain still poorly known, though. One of these rare complications is a peculiar larynx-shaped reorganization of the tracheal rings and cricoid cartilage. This tracheal narrowing seems to be nonsymptomatic and not life-threatening, at least as long as the reduction of the lumen is $<50 \%-75 \%$. Such a finding has never been described so far in the literature, and should be kept in mind as one of late complication of tracheostomy.
\end{abstract}

Keywords: Tracheostomy; Post-Tracheostomy Complications; Tracheal Stenosis; Optic Fiber Laryngoscopy; Double Larynx

\section{Introduction}

Tracheostomy is a common surgical procedure used in ENT and Intensive Care Units, consisting in the creation of an opening in the cervical trachea $\left(2^{\text {nd }}-3^{\text {rd }}\right.$ tracheal ring) to allow air to enter the lungs bypassing the upper airways. It is performed in election for airway protection (stroke, coma), for respiratory insufficiency (severe chronic obstructive bronchopneumopathy, neuromuscular diseases, upper airtract obstruction caused by laryngeal or esophageal cancers), and in emergency for immediate breathing assistance (acute respiratory insufficiency, unsuccessful intubation, facial trauma, foreign bodies, major burn).

The tracheostomy can be temporarily, and is performed when patients require long term respiratory support or are unable to protect their own airways: in this case, a tracheostomy tube is inserted to maintain the patency of the airway and can be removed when the patient recovers. On the other hand, a permanent tracheostomy is created where the trachea is brought out to the surface of the skin and sutured to the neck wall; this stoma is kept open by the rigidity of the tracheal cartilage; the patient breathes through this stoma for the remainder of his/her life. As a result, there is no connection between the nasal passages and the trachea.

Though it is mainly considered to be an "easy" procedure by most surgeons, tracheostomy has a number of consequences, that can be classified by the time of onset in intra-operative and post-operative complications. Acute intra-perioperative complications are hemorrhage, infection, subcutaneous emphysema, pneumothorax, tube obstruction, reflex arrhythmia, and accidental decannulation [1], while in the long run complications are represented by formation of granulation tissue, tracheal stenosis, tracheomalacia, tracheoinnominate-artery fistula, tracheoesophageal fistula, ventilator-associated pneumonia, and aspiration [2]. Up to $34 \%$ of late complications are represented by tracheal stenosis, only $6 \%$ of which are symptomatic [3]. Treatment depends on the nature and severity of the complication, from barely noticeable (subclinical granulation tissue) to life-threatening (major tracheal stenosis, acute hemorrhages of fistulae). Severely symptomatic cases require a surgical external (resection-anastomosis technique) or endoscopic (progressive dilatation by Montgomery T-tube, stent positioning, balloon, YAG or $\mathrm{CO}_{2}$ laser excision) approach.

In this paper, we report an unknown-so-far late complication of tracheostomy, characterized by a non-symptomatic post-operative larynx-shape rearrangement of the tracheal cartilage.

\section{Case Report}

A 60-year old male came to our ENT Clinic for progressive dysphonia in December 2007. After an extensive 
ENT exanimation, showing some white irregular lesions of the arytenoideal mucosa, and a subsequent laryngeal biopsy, he was dismissed with an histological diagnosis of granuloma of vocal processes of arythenoids and vocal folds dysplasia. In 2008 he had an heart attack and successfully underwent a coronary by-pass and received a temporarily tracheostomy for acute breathing assistance, which was removed two months later. In May 2011 he came back to our ENT clinic for a 3-month worsening hoarseness. He underwent ENT examination, a flexible optic fiber laryngoscopy, and a second laryngeal biopsy, that confirmed multiple bilateral granulomas of both arythenoid vocal processes, accounting for his symptoms.

Performing fibro endoscopy, the clinicians noticed an unusual narrowing below the glottis extending down to the $2^{\text {nd }}-3^{\text {rd }}$ tracheal ring, at the level of the previous tracheostoma. As documented by Figure 1 that shows shows a series of photographs, ordered in a reading-like progression from base of tongue to tracheal bifurcation level (from “ $A$ ” to " $F$ ”) in which, just below the true vocal folds it is possible to observe a peculiar, complex cartilaginous structure, containing a sort of supplementary epiglottis and even a second pair of true vocal folds. At a first glance, these cartilaginous rings, shaped in the fashion of "true vocal folds" and "epiglottis" mimicked perfectly a sort of second larynx, that macroscopically differs from the real one only for the absence of movement with phonation and breathing.

\section{Discussion}

Tracheostomy may cause a number of acute and late complications, from minimally symptomatic to life-threatening [2]. As mentioned above, complications can be classified in early or intra-perioperative (hemorrhage, infection, subcutaneous emphysema, pneumothorax, tube obstruction, reflex arrhythmia, and accidental decannulation [1]) and late or post-operative (formation of granulation tissue, tracheal stenosis, tracheomalacia, tracheoinnominate-artery fistula, tracheoesophageal fistula, ventilator-associated pneumonia, and aspiration [2]). Among them, tracheal stenosis, defined as an abnormal narrowing of the tracheal lumen, is the most common [4], needing surgical correction in 3\% - $12 \%$ of cases [5]. Instead, clinically significant benign stenosis occurs in about $1 \%$ of patients [6]. The most frequent types of stenosis are abnormal wound healing with excess formation of granulation tissue and web-like fibrosis at the stoma site [7]. A tracheal stenosis begins to produce symptoms when the lumen has been reduced by 50\% - 75\% [8].

In our case the patient developed a post-tracheostomy substenosis of the trachea that never reached $50 \%$ reduction of the normal lumen, thus justifying a complete absence of clinical manifestation, such as dyspnea or stridor,

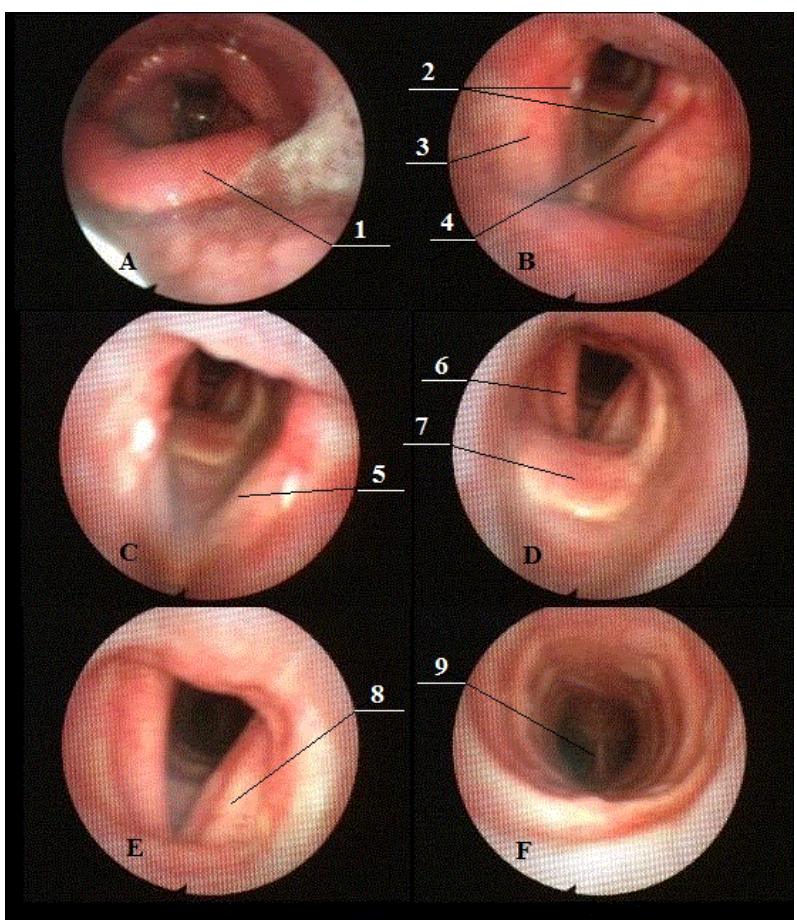

Figure 1. Opticfiber laryngoscopy stills ordered in a reading-like progression (from "A" to "F"), showing just below the true vocal folds a complex cartilaginous structure shaped in the fashion of a second larynx. 1: Epiglottis; 2: Granulomatosis of vocal processes of arythenoids; 3: Right false vocal fold; 4, 5: Left true vocal fold; 6, 8: "True vocal fold-shaped" tracheal stenosis; 7: “Epiglottis-shaped” tracheal stenosis; 9: Tracheal bifurcation.

probably due to the prolonged presence of an over-inflated cuff of the tracheostomy tube, impinging the anterior and lateral walls of the trachea. In fact, overinflation of a high-volume low-pressure cuff can lead to ischemic airway injury: with prolonged ischemia, mucosal ulceration, chondritis, and cartilaginous necrosis may ensue, leading to the formation of granulation tissue; the subsequent development of fibrosis and narrowing of the anterolateral aspects of the tracheal walls could be responsible for this unique kind of stenosis. Moreover, our patient had multiple risk factors known to be associated with stomal stenosis, including advanced age, male sex, tight-fitting or oversized cannula, prolonged placement, and disproportionate excision of anterior tracheal cartilage during the creation of the tracheostomy, as recently reported by Sue and Susanto [8].

To our knowledge, such a peculiar larynx-shaped reorganization of the tracheal rings and cricoid cartilage after a previous tracheostomy has not been described so far and should be kept in mind as one of the possible manifestations of post-tracheostomy substenosis, even though it doesn't lead to any anatomical or functional sequelae.

Our methods for informed consent, data analysis and 
follow-up were reviewed and approved by the Catholic University of the Sacred Heart Institutional Review Board, and are in accordance with the Helsinki Declaration.

\section{REFERENCES}

[1] D. Feller-Kopman, "Acute Complications of Artificial Airways," Clinics in Chest Medicine, Vol. 24, No. 3, 2003, pp. 445-455. doi:10.1016/S0272-5231(03)00047-9

[2] S. K. Epstein, "Late Complications of Tracheostomy," Respiratory Care, Vol. 50, No. 4, 2005, pp. 542-549.

[3] S. Norwood, V. L. Vallina, K. Short, M. Saigusa, L. G. Fernandez and J. W. McLarty, "Incidence of Tracheal Stenosis and Other Late Complications after Percutaneous Tracheostomy," Annals of Surgery, Vol. 232, No. 2, 2000, pp. 233-241. doi:10.1097/00000658-200008000-00014

[4] H. C. Grillo, D. M. Donahue, D. J. Mathisen, J. C. Wain and C. D. Wright, "Postintubation Tracheal Stenosis.
Treatment and Results," The Journal of Thoracic and CarDiovascular Surgery, Vol. 109, 1995, pp. 486-492. doi:10.1016/S0022-5223(95)70279-2

[5] J. M. Streitz and S. M. Shapshay, “Airway Injury after Tracheotomy and Endotracheal Intubation,” Surgical Clinics of North America, Vol. 71, 1991, pp. 1211-1230.

[6] M. Marel, Z. Pekarek, I. Spasova, et al., "Management of Benign Stenoses of the Large Airways in the University Hospital in Prague, Czech Republic in 1998-2003,” Respiration, Vol. 72, 2005, pp. 622-628.

[7] N. Zias, A. Chroneou, M. K. Tabba, et al., "Post Tracheostomy and Post Intubation Tracheal Stenosis: Report of 31 Cases and Review of the Literature,” BMC Pulmonary Medicine, Vol. 8, No. 18, 2008, pp. 18-26. doi:10.1186/1471-2466-8-18

[8] R. D. Sue and I. Susanto, "Long-Term Complications of Artificial Airways,” Clinics in Chest Medicine, Vol. 24, No. 3, 2003, pp. 457-471. doi:10.1016/S0272-5231(03)00048-0 